\title{
Hoarseness of Voice in a Patient with Posterior Fossa Tumor: A Diagnostic Error
}

\author{
Nidhi Singh ${ }^{1} \quad$ Kiran Jangra ${ }^{1, \odot ~ S h i v ~ L . ~ S o n i ~}{ }^{1}$ \\ ${ }^{1}$ Department of Anaesthesia and Intensive Care, Postgraduate \\ Institute of Medical Education and Research, Chandigarh, India \\ ${ }^{2}$ Department of ENT and Head Neck Surgery, Postgraduate Institute \\ of Medical Education and Research, Chandigarh, India \\ ${ }^{3}$ Department of Neurosurgery, Postgraduate Institute of Medical \\ Education and Research, Chandigarh, India
}

J Neuroanaesthesiol Crit Care 2022;9:64-65.

Airway assessment is occasionally difficult and challenging in neurosurgical situations, such as in unconscious and uncooperative patients, patients with a spinal fracture on traction, and patients with stereotactic frames in situ. An incomplete assessment may bring a surprise during laryngoscopy and intubation. Here, we present a case of posterior fossa space-occupying lesion (SOL) with hoarseness of voice where intubation of trachea became extremely challenging.

We report a case of 56 years old male with no known comorbidities who presented with chief complaints of swaying to the left side during walking for the past 3 months and headache for 1 month. His medical history was otherwise unremarkable. He was conscious but disoriented to time, place, and person and moved all limbs equally on examination. Gag and cough reflexes were weak, while other cranial nerve functions were intact. The patient had bradycardia (heart rate-56/min) and hypertension (blood pressure-170/100 $\mathrm{mm} \mathrm{Hg}$ ). Preoperative investigations including electrocardiography, chest radiograph, hemogram, random blood sugar (132 mg/dL), serum electrolytes (sodium-142 mmol/L, potassium-3.8 $\mathrm{mmol} / \mathrm{L}$ ), and renal functions (urea-28 $\mathrm{mg} / \mathrm{dL}$, creatinine- $0.9 \mathrm{mg} / \mathrm{dL}$ ) were within normal range.

Contrast-enhanced magnetic resonance imaging was suggestive of large, relatively well-defined heterogeneously enhancing, hemorrhagic intra-axial SOL in left cerebellum and vermis extending to the right side with mild surrounding perifocal edema, mass effect, and obstructive hydrocephalus ( - Fig. 1A). The provisional differential diagnosis included metastasis, hemangioblastoma, and glioblastoma.

The patient was posted for excision of the tumor. On preanesthetic examination, hoarseness of voice was noticed that

\author{
Address for correspondence Kiran Jangra, DM, Department \\ of Anaesthesia and Intensive Care, $4^{\text {th }}$ Floor, Nehru Hospital, \\ Postgraduate Institute of Medical Education and Research, \\ Chandigarh 160012, India (e-mail: drkiransharma0117@gmail.com).
}

was presumed to be due to the involvement of lower cranial nerves. As the patient was disoriented so, no further evaluation was done for hoarseness of voice. In the operation theater, standard anesthesia monitors were attached, and vitals were stable. Anesthesia was induced using morphine $7.5 \mathrm{mg}$ and titrated doses of propofol $(80 \mathrm{mg})$. Inj vecuronium $(7 \mathrm{mg})$ was given to facilitate the intubation of trachea. On direct laryngoscopy, an ulceroproliferative growth was observed on the left vocal cord with restricted mobility. An urgent consultation from an otolaryngorhinologist was sought, and a sample for biopsy was taken from the vocal cord growth (-Fig. 1B). Tracheal intubation was done using a smaller size endotracheal tube $(6.5 \mathrm{~mm})$ as presence of the growth precluded negotiation of a larger sized tube. The histopathological examination of the brain tumor sample revealed metastatic adenocarcinoma. The vocal cord lesion was found to be moderately differentiated squamous cell carcinoma. Tracheal extubation was done on postoperative day 1 , and the patient was discharged home on day 7 after complete evaluation for localizing the primary tumor.

After all investigations, we found that the patient had two different malignancies: adenocarcinoma metastasizing to the brain and squamous cell carcinoma at the vocal cord.

Brain metastases are more frequently encountered than primary brain tumors, occurring in $\sim 20$ to $40 \%$ of advanced-stage cancers. Lung, breast, melanoma (skin cancer), colon, and kidney cancers commonly spread to the brain. ${ }^{1,2}$ Hoarseness of voice is a usual patient complaint having a wide range of differential diagnosis, some benign and others quite advanced with significant morbidity. ${ }^{3}$ Broadly, the causes of hoarseness may be classified into neurological or non-neurological. Non-neurological causes include acute

published online October 17, 2021
DOI https://doi.org/ $10.1055 / \mathrm{s}-0041-1734420$ ISSN 2348-0548 (c) 2021. Indian Society of Neuroanaesthesiology and Critical Care. All rights reserved.

This is an open access article published by Thieme under the terms of the Creative Commons Attribution-NonDerivative-NonCommercial-License, permitting copying and reproduction so long as the original work is given appropriate credit. Contents may not be used for commercial purposes, or adapted, remixed, transformed or built upon. (https://creativecommons.org/licenses/by-nc-nd/4.0/).

Thieme Medical and Scientific Publishers Pvt. Ltd. A-12, 2nd Floor, Sector 2, Noida-201301 UP, India 

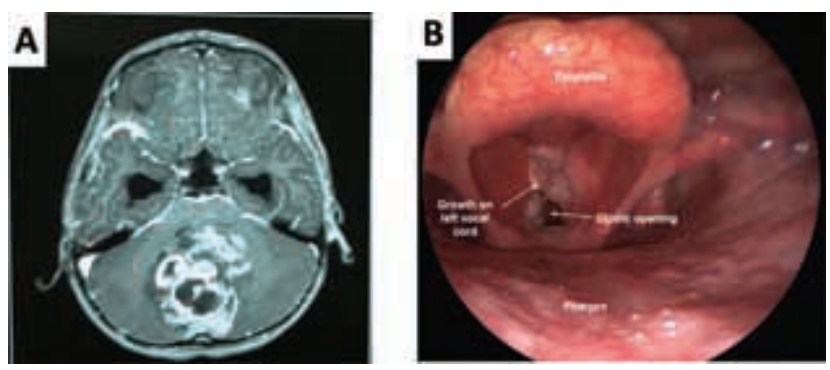

Fig. 1 (A) Axial section of post-contrast, T1-weighted, magnetic resonance imaging of brain showing heterogeneously enhancing space occupying lesion in the posterior fossa with mild perilesional edema and complete obliteration of the fourth ventricle. (B) Endoscopic view of vocal cords showing proliferative growth on left vocal cord involving the anterior commissure and causing narrowing the glottic opening.

and chronic laryngitis, misuse or overuse of the voice, gastroesophageal reflux, vocal nodules, polyps and cysts, vocal fold hemorrhage, vocal fold paralysis, physiological aging, or psychogenic factors. ${ }^{3.4}$

Neurological causes of hoarseness include vocal cord paralysis, multiple sclerosis, myasthenia gravis, Parkinson's disease, spasmodic dysphonia, essential tremor, muscle tension dysphonia, and vagus nerve injuries. ${ }^{3-5}$ Lesions of the vagus nerve may present with hoarseness alone due to paralysis of the vocal fold. ${ }^{5}$ Most of these etiologies of hoarseness of voice can be picked up using indirect laryngoscopy (IDL) and flexible fiberoptic bronchoscopy, which are noninvasive and handy tools.

Preanesthetic checkup of the patient in the preoperative period remains the cornerstone of safe anesthesia practice. ${ }^{6}$
Vocal cord malignancies usually do not metastasize to the brain. In this case, hoarseness of voice was due to vocal cord malignancy. However, it was overlooked as we presumed it to be due to lower cranial nerves involvement secondary to posterior fossa SOL.

Hence, we would like to emphasize that symptoms like mild hoarseness of voice should not be overlooked in the preoperative period and should be thoroughly evaluated using IDL or flexible fiberoptic bronchoscopy. As we do not have time for further evaluation in emergency scenarios, we must consider these patients as having difficult intubation and should be prepared accordingly. Another unique point about this case was the existence of two histopathologically distinct tumors at a different anatomical location in the same patient.

\section{Conflict of Interest}

None declared.

\section{References}

1 Seoane J, De Mattos-Arruda L. Brain metastasis: new opportunities to tackle therapeutic resistance. Mol Oncol 2014;8(6):1120-1131

2 Gállego Pérez-Larraya J, Hildebrand J. Brain metastases. Handb Clin Neurol 2014;121:1143-1157

3 Reiter R, Hoffmann TK, Pickhard A, Brosch S. Hoarseness-causes and treatments. Dtsch Arztebl Int 2015;112(19):329-337

4 Feierabend RH, Shahram MN. Hoarseness in adults. Am Fam Physician 2009;80(4):363-370

5 Erman AB, Kejner AE, Hogikyan ND, Feldman EL. Disorders of cranial nerves IX and X. Semin Neurol 2009;29(1):85-92

6 Zambouri A. Preoperative evaluation and preparation for anesthesia and surgery. Hippokratia 2007;11(1):13-21 